\title{
D-Space and Deform Closure Grasps of Deformable Parts
}

\author{
International Journal of Robotics Research, Vol 24(11), November 2005 \\ K. "Gopal” Gopalakrishnan and Ken Goldberg \\ IEOR and EECS Departments, UC Berkeley, USA \\ Contact: goldberg@ieor.berkeley.edu
}

\begin{abstract}
Building on the well-established form closure framework for holding rigid parts, this paper proposes a new framework for holding deformable parts. We consider the class of deformable parts that can be modeled as linearly elastic polygons with a triangular finite element mesh and given stiffness matrix. We define the $D$-space (deformation-space) of a part as the $C$-space of all its mesh nodes. Free space is the intersection of the set of topology-preserving mesh configurations with the complement of the union of all $D$ obstacles that represent collisions of the part with finger bodies. Consider a given set of finger bodies in frictionless contact with a part. When positive work is needed to release the part, we say that it is in deform closure. We present numerical examples and prove two results: (1) If a contact set holds a rigid part in form closure, it will hold the equivalent deformable part in deform closure and (2) deform closure is frame invariant.

We then consider frictionless deform closure grasps with two contact points. We define a measure of grasp quality based on balancing the potential energy needed to release the part against the potential energy that would result in plastic deformation. Given two jaw contacts at the perimeter nodes, we develop numerical algorithms to determine the optimal jaw separation based on this metric. For a part with $\boldsymbol{n}$ mesh nodes and $p$ perimeter nodes, we give an algorithm that computes the optimal separation in time $O\left(n^{3} p^{2}+p^{6} \log p\right)$ and an approximation algorithm that runs in time $O\left(n^{3} p^{2}+\left(p^{2} / \varepsilon\right) \log p\right)$.
\end{abstract}

Index Terms - Form Closure, Deform Closure, C-Space, D-Space, Finite Element Methods, Fixturing, Grasping, Workholding.

\section{Introduction}

Research on part-holding usually assumes that the parts are rigid solids [Bicchi00]. However, in many applications ranging from assembly to food handling to surgery to xerography, parts deform in response to applied forces. Because infinitesimal motion is unavoidable for deformable parts, the well-established models of first and second order immobility must be extended.

There is a substantial body of research on robotic holding (grasping and fixturing) of rigid objects. Our work combines FEM meshing with ideas from rigid body immobility by Rimon and Burdick [Rimon96] and caging grasps for rigid bodies by Rimon and Blake [Rimon99].

In our model, the part perimeter behaves like a planar structure whose perimeter is composed of struts of variable length, with hinges at each vertex. We define the D-space (deformation-space) of the part as the Cspace of all nodes in its mesh. Given the initial configuration $q_{0}$ of the mesh in D-space, we define $D_{T}$ as the set of all configurations with the same mesh topology as $q_{0}$. We consider contact with a set of rigid finger bodies $A=\left\{A_{1}, \ldots, A_{k}\right\}$. Each finger defines a D-obstacle $D A_{i}$, the set of mesh configurations that collide with $A_{i}$. The free space $D_{\text {free }}$ is the set of feasible configurations, the intersection of $D_{T}$ with the complement of the union of D-obstacles.

Given part $E$, mesh $M$, FEM stiffness matrix $K$, and contact set $A$, we can determine the potential energy of any point in $D_{\text {free }}$. Given a candidate configuration $q_{A}$, we say that the part can be released from $A$ by a sequence of external wrenches if the part can escape from $q_{A}$. We define $U_{A}$ as the minimum work that needs to be done by these wrenches to release the part from $A$. We say that $A$ holds $E$ in deform closure if $U_{A}>0$.

We then consider the case where two fingers or jaws make point contact with a given polygonal part at a given pair of mesh nodes. We define a new quality metric that balances between squeezing too tight and not tight enough. The metric is maximized when the work needed to release the part is equal to the work needed 
to cause plastic (irreversible) deformation of the part. Note that two point contacts can hold a part in deform closure just as two point contacts at concavities can hold a part in form closure [Gopalakrishnan02].

As intuition behind our algorithm for computing the optimal jaw separation, we first consider only release paths that involve minimal increase in potential energy. This allows us to limit our attention to only configurations where both jaws are in contact with the part. Thus, for a given jaw separation, we can represent the potential energy as a function of the positions of both jaws along part perimeter (represented by arclength parameters). Thus, the potential energy is a two-dimensional surface embedded in three dimensions. It is zero when the jaws are relaxed and non-zero when the jaws deform the part. We can further simplify the problem by considering only discrete points on this potential energy surface. We represent these points in a "contact graph", where each graph vertex corresponds to a pair of mesh nodes. A release path through $D_{\text {free }}$ corresponds to a sequence of adjacent vertices on the contact graph, starting at the vertex corresponding to the given deform closure grasp and ending at any other vertex corresponding to an equilibrium grasp for that value of jaw separation. We develop an algorithm inspired by Dijkstra's algorithm to determine the potential energy required to reach an equilibrium configuration.

The potential energy required to release the part as a function of jaw separation is continuous and piecewise differentiable. Our exact algorithm exploits the nature of the quality metric function to identify critical points that help determine the optimal jaw separation. Our approximate algorithm computes the value of the function by uniform sampling, using a sampling interval based on the stiffness coefficients and a given approximation bound. The sample point with the maximal value of the objective function provides an $\varepsilon$-approximation to the optimal jaw separation.

\section{Related Work}

Bicchi and Kumar provide a concise survey of literature on grasping and fixturing in [Bicchi00]. Grasps of rigid bodies can be classified as force or form closure. Form closure (immobility) occurs when any neighboring configuration of the part results in collision with an obstacle. Force-closure occurs if any external wrench can be resisted by applying suitable forces at the contacts [Mason01, Rimon98]. Gripper contacts can be modeled as frictional points, frictionless points or soft contacts [Salisbury82, Kim03].

The mobility of rigid bodies in contact with frictionless finger bodies was initially studied using first order approximations [Realeaux1876, Somoff1900, Mishra87, Markenscoff90]. The first order theories are based on approximations of part geometry in infinitesimal neighborhoods and part motion of an infinitesimal length. However, these first order approximations of mobility do not always predict immobility correctly.

Rimon and Burdick [Rimon96] give rigorous definitions of first and second order immobility. They express paths of the part in free space using functions $q(t)$ based on scalar parameter $t$. They consider the distances $d_{i}$ from the $i^{\text {th }} \mathrm{C}$-obstacle surface to $q(t)$. They then consider the first and second order terms in the algebraic expressions of distances as a function of $t$. The second order terms are needed if first order tests do not show if the distance is increasing or decreasing (the derivative at $t=0$ is 0 ). Geometrically speaking, the first order approximation of immobility is equivalent to approximating the trajectory of the part in Cspace as a straight line, and the surfaces of the C-obstacles as hyperplanes. Second order immobility is equivalent to approximating the trajectory as an elliptical arc and the C-obstacle surfaces as ellipsoids.

Rimon and Blake [Rimon99] give a method to find caging grasps, configurations of jaws that constrain parts in a bounded region of $\mathrm{C}$-space such that actuating the gripper results in a unique final configuration. They consider the opening parameter of the jaws as a function of jaw positions along the perimeter of the part and use stratified Morse theory to find caging grasps by finding limiting cases that occur when the opening parameter is at a saddle point. [Gopalakrishnan02] also uses the distance function to determine immobile grasps of 2D polygonal parts by a pair of vertical cylindrical jaws engaging the part at its concave vertices. Jia [Jia04] gives a fast algorithm to compute all grasps at pairs of antipodal points of a curved part. 
The algorithm divides the part into concave and convex pieces at points of inflexion and numerically examines these pieces by bisection in order to compute the grasps.

An efficient geometric algorithm to compute all placements of four frictionless point contacts on a polygonal part that ensure form closure is described by van der Stappen et al [vanderStappen99]. Given a set of four edges, they show how to compute critical contact placements in constant time. The time complexity of their algorithm is bounded by the number of such sets, and runs in an expected time of $O\left(n^{2} \log n\right)$ for $n$ vertices. Cheong et al [Cheong03] give fast algorithms to find immobilizing grasps of 2D polygonal parts with 2 and 3 contacts. The algorithms find sets of contact wrenches that contain the center of mass. Zhu et al [Zhu03] give a grasp metric for 2D and 3D grasps to quantify how firmly the rigid part is held when resisting external forces. Their metric is faster to compute than similar earlier metrics.

Cheong et al [Cheong02] give upper bounds on the number of point contacts needed to hold a chain of polygons hinged at connecting vertices. They consider the centers of rotation for each polygon such that the hinge vertices have the same linear velocities for the pair of polygons they connect. They place contacts such that no such rotation is possible. Milgram and Trinkle [Milgram02] describe the topology of C-spaces of mechanisms that consist of chains of links connected by rotational joints.

[Ramamurti98] gives an excellent review of finite element methods and speed and memory optimizations for numerical solutions of FEM problems. To solve an FEM problem with $n$ elements exactly, $O\left(n^{3}\right)$ time is required. The numerical approximation makes use of the fact that FEM stiffness matrices are sparse. As a result, there are only $O(n)$ non-zero entries in a matrix with $n^{2}$ elements. [Corman01] presents several graph algorithms and data structures. Of particular interest to our algorithm is the proof of correctness and time complexity of the modified Dijkstra's algorithm for sparse graphs implemented with binary heaps. For a graph with $n$ vertices and $O(n)$ edges, the shortest path can be computed in $O(n \log n)$ time.

Recent work on fixturing deformable and sheet-metal parts is based on the work of Menassa and De Vries [Menassa91] where they determine the positions of the primary datum (the datum points needed to locate the part in the correct plane) for 3-2-1 fixturing to minimize deformation. They use a finite element model of the part, and determine fixture locations by optimizing an objective that is a function of the deformations at the nodes. Their work is extended by [Rearick93] and [Cai96].

Gopalakrishnan et al [Gopalakrishnan03, Gopalakrishnan04c] propose unilateral fixtures, a new class of fixtures for sheet metal parts with holes, where holding elements lie almost completely on one side of the part, maximizing access for welding, assembly, or inspection. Each primary jaw is cylindrical with a conical groove that provides the equivalent of four point contacts and facilitates part alignment. They present a two-phase algorithm for computing unilateral fixtures where the second phase uses a Finite Element Method (FEM) to compute part deformation and to arrange secondary contacts at part edges and interior surfaces. For a given sheet-metal part, given as a 2D surface embedded in 3D with $e$ edges, $n$ concavities and $m$ mesh nodes, Phase I takes $O\left(e+n^{4 / 3} \log ^{1 / 3} n+g \log g\right)$ time to compute a list of $g$ pairs of primary jaws ranked by quality. Phase II computes the location of $r$ secondary contacts in $O\left(g m^{3} r\right)$ time.

Wakamatsu et al [Wakamatsu96] extend the concept of force closure for rigid parts with unbounded applied forces to deformable parts with bounded applied forces. They consider a candidate grasp and external forces within a bound that can deform and displace the part. They assume that the contact normals do not vary as forces are applied, but allow contacts to be broken. They define "bounded force closure" as grasps that can resist any external force within the bound.

Howard and Bekey [Howard97] model deformable parts using a spring-mass model and use a neural network to control a gripper. They use tactile feedback to learn the properties of the deformable parts, and thus determine the minimum force needed to lift the deformable part. Heinrich and Worn's edited collection of papers [Heinrich00] describes deformation models and control algorithms for the manipulation of deformable objects. These include the modeling of fabrics, continuous modeling of deformation of linear objects such as beams, collaborative manipulation of deformable parts, laying out wires and cables and sewing. [Lin00] study the dual problem of holding a part with compliant fingers. They consider frame- 
invariant parameters derived from the stiffness to compute a metric for multi-fingered grasps and fixtures. They use this metric to derive optimum three and four fingered fixtures.

Hirai et al [Hirai01] propose a robust control law for the manipulation of deformable parts. They use tactile feedback from the actuators and video feedback for the positions of selected reference points to control the motion of a deformable part. Li et al [Li02] design fixtures for laser welding using a genetic algorithm within robust design spaces with low to part dimension and jaw location errors. Li et al [Li02b] describe a dexterous part holding mechanism based on vacuum cups and model the elastic deformation of the sheet-metal part using Finite Element Methods and a statistical data model. The results from this model are used to minimize part deformation. Shiu et al [Shiu97, Shiu03] give a heuristic algorithm to analyze the deformation of a sheet metal part by decoupling it into beams based on part features, and give an algorithm to allocate tolerances to each feature.

Path planning for elastically deformable parts has been studied using probabilistic roadmaps (PRM). Holleman, Kavraki and Warren [Holleman98] give a path planning algorithm for a flexible surface patch. They use a Bezier approximation and an approximate energy function to model deformation of the part. They present experimental results of paths planned for parts generated by a search graph using PRM. Guibas et al [Guibas99] improve on the PRM methods for path planning for a surface patch by studying the medial axis of the workspace. They then compute minimum energy configurations of the part for positions along the axis that are connected by quasi-static paths. Lamiraux et al [Lamiraux99] generate a path for a thin rectangular elastic metal plate represented by a Bezier when it is manipulated by constraining the positions and orientations of two opposite edges. Given the controlling constraints, the shape of the plate is computed by minimizing the potential energy due to the deformations. They generate paths using PRM on the configurations of the edges being controlled. Moll and Kavraki [Moll04] have extended this approach to systems such as surgical sutures and snake robots by computing configurations using constrained minimization of potential energy with the length of the curve fixed. However, if there is contact between the plate and an obstacle, they discard the configuration. Bayazit et al [Bayazit02] extend this by approximating the deformations of the part due to the obstacles for fast computation. For the simplest approximation, they consider the deformation of the bounding box of the part. Better approximations include moving the part mesh away from the obstacles along the outward facing normals, or sample possible deformations along directions based on normals of the part or the obstacles.

This work is a revised and extended version of material presented in [Gopalakrishnan04] and [Gopalakrishnan04b].

\section{Deformation-Space and Deform Closure}

\subsection{Deformation-Space}

Consider an FEM model with linear interpolation and linear elasticity. The input is a polygonal part $E$ with polygonal holes, modeled by a 2D triangular FEM mesh $M$, and the initial undeformed configuration $q_{0}$ of the part, defined in a global reference frame. In this model, the perimeter behaves like a polygon with variable length edges and hinges at each vertex.

We formally define the Deformation Space (D-Space) of the part as the C-Space of its mesh nodes. We then characterize the sub-space of topology-preserving and collision-avoiding deformations. Although we focus on cases with only planar translational degrees of freedom, the general definition is as follows. Depending on the FEM model used, each of the $n$ nodes in $M$ has a predefined number $f$ of degrees of freedom consisting of translational $(t$ DOFs) and rotational/torsional ( $r$ DOFs). Every mesh configuration is specified by specifying each of the $f$ freedoms of each of the $n$ nodes. Given the positions and geometries of all fingers, we represent the mesh in its deformation-space, the smooth manifold $D=\boldsymbol{R}^{t} \times \boldsymbol{R}^{r}$ when rotations are parameterized by $\theta \in \boldsymbol{R}^{r}$ via exponential map parameterization. Thus, for a part with $n$ nodes in $M$, each with $f$ degrees of freedom each, the D-space can be parametrized by $\boldsymbol{R}^{n f}$, with each coordinate axis 
corresponding to one DOF of one FEM node. We can represent any deformed translated or rotated shape of this part as a unique point in this space. For any point $q$ in $D$, we denote the corresponding shape and configuration of the part by $E(q)$. In our examples with triangular elements with linear elasticity and linear interpolation, $r=0$ and $t=2$.

We define $D_{T}$ as the set of all $q$ that preserve the topology of $M$ identical to the topology at the given undeformed configuration $q_{0}$. Given a finger body $A_{i}$, its corresponding D-space obstacle $D A_{i}$ is the set of all configurations $q$ such that $E(q)$ intersects $A_{i}$.

We define $D_{\text {free }}=\left(\bigcup_{i=1 . . k} D A_{i}\right)^{C} \cap D_{T}$ as the set of all valid deformable configurations of $M$.

Fig. 2 illustrates examples of configurations that lie in $D_{T}$ and $D_{T}^{C}$. Fig. 3 shows slices of $D_{\text {free }}$ for a rectangular part with 5 nodes and a small circular finger body (Fig. 3(a)). Each node has 2 translational degrees of freedom along the $\mathrm{x}$ and $\mathrm{y}$ axes. Fig. 3(b) assumes nodes 1-4 are fixed, and shows the resulting $D_{\text {free }}$ for node 5. Fig. 3(c) assumes nodes 1, 2, 4 and 5 are fixed, and shows $D_{\text {free }}$ for node 3.

\subsection{Potential Energy in D-Space}

We include the mechanical properties of the part by using the potential energy of any deformed state as determined by an FEM model. We assume zero friction between the part and finger contacts, and that the part exhibits perfect linear elasticity with known Young's modulus and Poisson's ratio. The parameters and the mesh geometry define the stiffness matrix, $\mathrm{K}$, that we give as input.

Let $X$ be the vector of displacements of all nodes from their initial positions in configuration $q_{0}$, expressed in the global coordinate frame used to define the FEM model ( $M, K, X$ ). In FEM, $X$ is usually referred to as the nodal displacement vector. $X$ is a distance preserving transformation of $q$ and a special case of expressing $q$ when we express nodal degrees of freedom relative to a reference frame whose origin coincides with the mesh node at configuration $q_{0}$, and whose axes are parallel to the axes of the global coordinate frame. Thus, the deformation potential energy is a scalar that is a function of the deformation of each node. The potential energy at any configuration $q$ in $D_{\text {free }}$ is represented as $U(q)=(1 / 2) X^{T} K X$. The potential energy is invariant to rigid transformations. The stiffness matrix $K$ is a positive semi-definite matrix $K$ such that for displacements $X$ representing rigid body transformations of $E, X^{T} K X=0$, and for $X$ representing deformations of the part $E, X^{T} K X>0$.

\subsection{Deform Closure}

To define deform closure, we consider as input: 1. the FEM mesh $M$ of the part $E$, 2. stiffness matrix $K$ of the part $E$ corresponding to mesh $M, 3$. the initial configuration $q_{0}$ of the mesh that specifies the initial topology and undeformed mesh shape, 4. set $A$ of frictionless contacts (finger bodies) that represents the grasp/fixture, and 5. the configuration $q_{A}$ in which the part is held by $A$.

We propose to characterize deform closure configurations of $E$ based on the D-Space model with potential energy. Note that we use symbols with different subscripts to different but related concepts. For example, $\mathrm{U}$ is used to represent potential energy, while $U_{A}\left(q_{A}\right)$ is used to represent the additional potential energy needed to release a part.

Definition: An equilibrium configuration is any configuration at a local minimum of the potential energy in $D_{\text {free. }}$ In the absence of friction and inertial forces, the part comes to rest at an equilibrium configuration.

Definition: We consider a set of wrenches that act on $E\left(q_{A}\right)$ for any equilibrium configuration $q_{A}$. If after the wrenches are applied and then removed, the part may not return to $q_{A}$, the wrenches are said to release E.

Definition: If $E\left(q_{A}\right)$ is held by $A$ such that no set of wrenches that increases the deformation potential energy of $E$ by at most $U$ can release $E$ from $A$, we say $q_{A}$ satisfies the property stable $(U)$. 
In other words, given $U \geq 0$, A holds $E\left(q_{A}\right)$ in stable $(U)$ if and only if $1 . q_{A}$ is at a local minimum of the potential energy in $D_{\text {free }}$, and 2. We consider all continuous subsets $S$ of $D_{\text {free }}$ such that for all $q_{s} \in S$, $U\left(q_{s}\right) \leq U\left(q_{f}\right)+U$. For all such subsets $S$, there should not exist $q_{1} \in S$ distinct from $q_{A}$ with $q_{1}$ at a local minimum of the potential energy in $D_{\text {free }}$.

Definition: The threshold potential energy $U_{A}$ for $A$ holding $F$ in configuration $q_{A}$ is defined as $U_{A}\left(q_{A}\right)=\sup \left\{U \mid A\right.$ holding $E\left(q_{f}\right)$ satisfies stable $\left.(U)\right\}$ if $q_{A}$ is an equilibrium configuration, and 0 otherwise. A holds $E\left(q_{A}\right)$ in deform closure if and only if $U_{A}\left(q_{A}\right)>0$.

A configuration with potential energy $U\left(q_{A}\right)+U_{A}\left(q_{A}\right)$ where the part can be released from the deform closure configuration is called its escape configuration. This is inspired by Rimon and Blake's analysis of caging grasps [Rimon99]. They define a cage of a part $E$ at configuration $q$ by a k-fingered hand with opening parameter $\sigma$. A configuration $x=(q, \sigma)$ of the part and the hand is a cage if $q$ lies in a connected component of free space $C_{\text {free }}$ which is completely surrounded by the finger C-obstacles $C A_{1}, \ldots C A_{k}$. They define the caging set $C$ as the set of all hand configurations that maintain the part caged between the fingers such that from any initial configuration in $C$, there exists a continuous path in $C$ leading to a desired immobilizing grasp $x_{0}=\left(q_{0}, \sigma_{0}\right)$. They use stratified Morse theory to analyze cages, and identify maximal caging grasps or puncture grasps as occurring at configurations where the distance between the fingers is at a saddle point.

Similarly in D-space, given a configuration $q_{A}$ that is a strict local minimum of the potential energy, we would like to identify the maximum $U$ for which A holds $E\left(q_{A}\right)$ in deform closure, by identifying escape configurations near $q_{A}$. This value of $U_{A}$ can be used to define a metric for the deform closure configuration.

\subsection{Frame Invariance and Equivalence Theorems}

Given a rigid part, we call an undeformed deformable part with the same shape and configuration of the rigid part its equivalent deformable part.

Theorem 1: If A holds a rigid part in form closure, A will hold the equivalent deformable part in deform closure, and vice versa.

By equivalent, we mean that the undeformed part has the same shape and configuration as the rigid part.

Proof: Consider the rigid part in an immobile configuration is immobile, we need to deform the deformable part in order to perturb it. Since this increases the deformation potential energy, $U_{A}>0$.

Consider a deformable part in deform-closure. Since deform-closure is a strict local minimum of potential energy, without increasing the potential energy, it cannot be moved without deforming it. Thus, the rigid part cannot be moved from the fixture too. This theorem also holds for higher orders of immobility.

Theorem 2: Frame Invariance.

The definition of deform closure is frame invariant, i.e. it does not depend on the global reference frame used.

Since the definition of $D$-space changes with the reference frame used, we prove that the definition of deform closure fixtures does not change with the global reference frame by showing the following:

(a) Displacements caused by a given force are frame invariant

(b) Potential Energy due to a given set of displacements is frame invariant, and

(c) A set of configurations of the part whose image is continuous in a $D$-space defined using one global reference frame has an image that is continuous in global in a D-space defined using any other reference frame.

Proof: We denote the global stiffness matrix in reference frame $g$ as $K_{g}$. For mesh element $\mathrm{m}_{\mathrm{i}}$, we denote the component of the stiffness matrix $K$ as $K_{i}$, the transformation matrix between the reference frame of the 
element and the global reference frame $g$ by ${ }_{g} T_{i}$, and the stiffness of element $\mathrm{m}_{\mathrm{i}}$ in the local frame by $K_{i}$. Thus, the stiffness matrix $K$ is the sum of its components for each mesh element and is given by:

$$
\begin{aligned}
& K_{g}=\sum_{i} K_{i} \\
& =\sum_{i}{ }_{g} T_{i} \cdot K_{i} \cdot{ }_{g} T_{i}^{T} .
\end{aligned}
$$

We denote the two reference frames by $g=1$ and 2 , and the transformation matrix between the frames by ${ }_{2} T_{1}$. The stiffness matrix changes with the frame used. The two stiffness matrices are related to each other as follows:

$$
\begin{aligned}
& { }_{2} T_{i}={ }_{2} T_{1} \cdot{ }_{1} T_{i} \\
& \Rightarrow K_{2}=\sum_{i}{ }_{2} T_{i} \cdot K_{i} \cdot{ }_{2} T_{i}^{T} \\
& =\sum_{i}{ }_{2} T_{1} \cdot{ }_{1} T_{i} \cdot K_{i \cdot 1} T_{i}^{T} \cdot{ }_{2} T_{1}^{T} \\
& ={ }_{2} T_{1}\left(\sum_{i}{ }_{1} T_{i} \cdot K_{i \cdot 1} T_{i}^{T}\right){ }_{2} T_{1}^{T} \\
& ={ }_{2} T_{1} \cdot K_{1} \cdot{ }_{2} T_{1}^{T}
\end{aligned}
$$

To prove (a), we consider any given set of forces expressed in reference frame 1 by vector $F_{1}$. Thus:

$$
X_{1}=K_{1}^{-1} \cdot F_{1} \text {. }
$$

The forces expressed in frame 2 are:

$$
F_{2}={ }_{2} T_{1} \cdot F_{1}
$$

The displacements predicted by frame 1, when transformed to reference frame 2 are:

$$
\begin{aligned}
& X_{2}={ }_{2} T_{1} \cdot X_{1} \\
& ={ }_{2} T_{1} \cdot K_{1}{ }^{-1} \cdot F_{1} \\
& ={ }_{2} T_{1} \cdot K_{1}^{-1} \cdot{ }_{2} T_{1}^{-1} \cdot{ }_{2} T_{1} \cdot F_{1} \\
& =\left(\left({ }_{2} T_{1}\right)^{T}\right)^{-1} \cdot K_{1}{ }^{-1} \cdot{ }_{2} T_{1}^{-1} \cdot{ }_{2} T_{1} \cdot F_{1} \\
& =\left({ }_{2} T_{1} \cdot K_{1} \cdot{ }_{2} T_{1}^{T}\right)^{-1} \cdot\left({ }_{2} T_{1} \cdot F_{1}\right) \\
& =K_{2}{ }^{-1} \cdot F_{2}
\end{aligned}
$$

This is the displacement predicted by reference frame 2 . Thus, (a) is true.

To prove (b), we consider the potential energy predicted by the reference frame 1 when $E$ is subject to deformations $X_{1}$ is $1 / 2 X_{1}^{T} K_{1} X_{1}$.

The potential energy predicted in frame 2 when $E$ is subject to the same deformation (but transformed to frame 2) is:

$$
\begin{aligned}
& 1 / 2 X_{2}^{T} \cdot K_{2} \cdot X_{2} \\
& =1 / 2\left({ }_{2} T_{1} \cdot X_{1}\right)^{T} \cdot\left({ }_{2} T_{1} \cdot K_{1} \cdot{ }_{2} T_{1}^{T}\right) \cdot\left({ }_{2} T_{1} \cdot X_{1}\right) \\
& =1 / 2 X_{1}^{T} \cdot{ }_{2} T_{1}^{T} \cdot{ }_{2} T_{1} \cdot K_{1} \cdot{ }_{2} T_{1}^{T} \cdot{ }_{2} T_{1} \cdot X_{1} \\
& =1 / 2 X_{1}^{T} \cdot K_{1} \cdot X_{1}
\end{aligned}
$$

This is the same as the potential energy in frame 1. Thus, (b) is true.

To prove (c), we show that for any two configurations of the part, the distance between their images in $D$ space remains the same in all frames of reference, which implies equivalence in continuity in both frames of reference. 
$\left|q_{a}-q_{b}\right|=\sum\left(\left(x_{n a}-x_{n b}\right)^{2}+\left(y_{n a}-y_{n b}\right)^{2}\right)$, summed over all mesh nodes $n$, where $x_{n i}$ and $y_{n i}$ are the $\mathrm{x}$ and y co-ordinates of node $n$ in configuration $i=a$, $b$. Since we consider only distance-preserving transformations when changing reference frames, $\left(\left(x_{n a}-x_{n b}\right)^{2}+\left(y_{n a}-y_{n b}\right)^{2}\right)$ is frame invariant for all nodes $n$. Thus, $\left|q_{a}-q_{b}\right|$ is also frame-invariant.

\section{Optimal Two-Point Deform-Closure Grasps}

\subsection{Problem Description}

We now consider two-point grasps: where there are exactly two jaws (finger bodies that make point contact). We specify the initial placement of these jaws on two mesh nodes $n_{0}$ and $n_{1}$. The jaw separation, $\sigma$, is the Euclidean distance between the jaw contact points. In what follows, we describe our approach in terms of contracting grasps (where the contacts exert forces towards each other). An analogous approach can be developed for expanding grasps.

We define a quality metric $Q$ to evaluate deform-closure grasps. We define $\sigma^{*}$ as the jaw separation that maximizes $Q: Q\left(\sigma^{*}\right)=\max _{\sigma}\{Q(\sigma)\}$. Note that $U_{A}$, the threshold potential energy, is not an appropriate quality metric because for many parts we would trivially close the jaws to zero separation to maximize it.

We introduce the elastic limit of part material to define a metric that balances the gripping force against forces that will result in plastic deformation of the part. Given the elastic limit strain $e_{L}$ of the part material, we consider the jaw separation $\sigma_{L}$ at which at least one triangular mesh element reaches the elastic limit. We define $U_{L}(\sigma)$ as the difference between the potential energies at separations $\sigma$ and $\sigma_{L}$. We define:

$$
Q(\sigma)=\min \left\{U_{A}(\sigma), U_{L}(\sigma)\right\} .
$$

At the optimal jaw separation $\sigma^{*}$, the potential energy needed to release the part equals the potential energy needed to squeeze it to its elastic limit.

\subsection{Problem Description}

We develop an algorithm to determine $\sigma^{*}$.

Input: Polygonal part $E$, mesh $M$ with $n$ mesh nodes and $p$ perimeter nodes, stiffness matrix $K$, initial undeformed configuration $q_{0}$, initial contact mesh nodes $n_{0}$ and $n_{l}$, and elastic limit strain $e_{L}$ (Fig. 5).

Output: Optimal jaw separation $\sigma *$ that maximizes $Q(\sigma)$.

We make the following assumptions:

1. The perimeter of the part does not collide with itself at intermediate configurations as it deforms from the deform-closure grasp to the escape configuration.

2. Mesh triangles do not become degenerate. We assume we are given a sufficiently dense mesh that does not result in degeneracy.

3. If we place one contact on the perimeter and move the other along the perimeter at a fixed separation from the first contact, the local maxima and minima of potential energy occur when the second contact is at a mesh node. Strictly speaking, given a particular mesh, the actual potential energy may be a maximum or a minimum even when the contact is at a mesh edge (but not at a mesh node), with the line joining the contacts being perpendicular to the edge. However, given a sufficiently dense mesh (with more mesh nodes near the contact), the difference between the maximum potential energy and the potential energy with the second contact at a vertex can be shown to converge to zero as mesh density increases, under the assumption that the error in the FEM approximation of the part also converges to zero. 
We explain our approach to solve this problem in detail in the following sections. In brief, consider the curve $Q(\sigma)$ which is continuous and piecewise differentiable. For the exact, algorithm, we identify sampling points on the curve based on end-points of intervals where the curve is differentiable. We evaluate the quality at these sampling points to determine the exact function $Q(\sigma)$. For the approximate algorithm, we bound the slope at any point on the curve. This bound is used to generate uniformly-spaced sampling points where we evaluate $Q(\sigma)$ such that the error in $Q\left(\sigma_{\varepsilon}\right)$ due to sampling is at most $\varepsilon$. In order to bound the slope and in order to determine $Q(\sigma)$ at the sampling points, we need to first identify points and paths of interest as the part is released with minimum increase in potential energy. Generating these points and their potential energies for a given separation allows us to compute the threshold potential energy for a given jaw separation.

\subsection{Points and Paths of Interest in D-Space}

To compute $\sigma^{*}$, we would like to determine a path that the part can follow to be released with minimal increase in potential energy. Since it is inefficient to consider every possible path in D-space, we create a graph to represent key configurations as graph vertices.

We use the following notation: $q_{A}$ is the initial configuration of the part when grasped by contacts at $(0,0)$ and $(\sigma, 0)$ engaging the part at $n_{0}$ and $n_{l}$ respectively. $\sigma\left(n_{a}, n_{b}\right)$ is the distance between mesh nodes $n_{a}$ and $n_{b} . \sigma_{0}\left(n_{a}, n_{b}\right)$ is the distance between mesh nodes $n_{a}$ and $n_{b}$ at configuration $q_{0}$.

To release the part from $A$, we move it along a path in D-space starting at $q_{A}$ and ending at an escape configuration. To determine the threshold potential energy, we are interested in an escape configuration with least potential energy. From assumption 3, if one contact were fixed on the part perimeter and the other jaw is allowed to slide along the perimeter, the potential energy as a function of the position of the second jaw is monotonic (increasing or decreasing) in every interval corresponding to one mesh edge. This implies: 1) there exists such an escape configuration $q_{p}$ with both contacts at mesh nodes, and 2) there exists a path in D-space from $q_{A}$ to $q_{p}$ such that one of the contacts is always at an FEM node such that $q_{p}$ has the maximum potential energy among all configurations on the path. It is sufficient to consider the paths where one contact is always at a mesh perimeter node.

We construct a "contact graph" $G$ to represent such paths. Let $V$ be the set of vertices in $G$. Every graph vertex $v\left(n_{a}, n_{b}\right)$ corresponds to point contacts present at a pair of perimeter mesh nodes $n_{a}$ and $n_{b}$. These are points of interest in D-Space. We connect graph vertices $v\left(n_{a}, n_{b}\right)$ and $v\left(n_{a}, n_{c}\right)$ are connected by an edge if: 1) $n_{b}$ and $n_{c}$ are connected by a mesh edge on the part perimeter, or 2) an arc centered on $n_{a}$ and having radius $\sigma$ and lying on the exterior of the part has one end point $n_{b}$ and the other end-point on a mesh edge flanking $n_{c}$. The second case is when the part can be rotated about a mesh node till the contact reaches the perimeter near another mesh node. Paths in this graph represent paths of interest in D-Space.

\subsection{Potential Energies at Graph Vertices}

At each graph vertex, we are interested in the minimum possible potential energy when the contacts engage the part at the corresponding mesh nodes $n_{a}$ and $n_{b}$, with separation $\sigma$. This is determined by solving the FEM model with boundary conditions restricting $n_{a}$ at $(0,0)$ and $n_{b}$ at $(\sigma, 0)$.

We note that we use a linear FEM system to model the part. We compute nodal deformations by solving a system of linear equations subject to the boundary conditions. As a result, for any graph vertex when the two contacts perform a contracting grasp at $\left(n_{a}, n_{b}\right)$, the potential energy associated with the corresponding vertex is a quadratic function of $\sigma$ given by $U\left(n_{a}, n_{b}, \sigma\right)=1 / 2 k_{a b}\left(\sigma-\sigma_{0}\left(n_{a}, n_{b}\right)\right)^{2}$, when the jaws squeeze the part, and 0 when the jaws are relaxed. Here, $k_{a b}$ is the component of the force applied by the contacts towards each other when $\sigma_{0}\left(n_{a}, n_{b}\right)-\sigma=1$ unit. However, for $\sigma>\sigma_{0}\left(n_{a}, n_{b}\right)$, the potential energy for contracting grasps is 0 . It is equivalent to replacing the part by a linear spring of stiffness $k_{a b}$. 
We first compute the value of $k_{a b}$ for all $v\left(n_{a}, n_{b}\right)$ by solving the FEM model with boundary conditions $n_{a}$ is at $(0,0)$ and $n_{b}$ is at $\left(\sigma_{0}\left(n_{a}, n_{b}\right)-1,0\right)$.

For the graph vertex $v\left(n_{0}, n_{1}\right)$ alone, we also compute the strains at each mesh node. We find the maximum strain over all nodes as $e_{\max }$.

Thus each graph vertex $v\left(n_{a}, n_{b}\right)$ is associated with a potential energy $U\left(n_{a}, n_{b}, \sigma\right)=1 / 2 k_{a b}\left(\sigma-\sigma_{0}(\right.$ $\left.\left.n_{a}, n_{b}\right)\right)^{2}$, where $k_{a b}$ are known. Some of these vertices correspond to equilibrium configurations. A vertex $v$ $\left(n_{a}, n_{b}\right)$ is in equilibrium if for every neighbor $v\left(n_{c}, n_{d}\right)$ of $v\left(n_{a}, n_{b}\right)$,

$$
U\left(n_{c}, n_{d}, \sigma\right) \geq U\left(n_{a}, n_{b}, \sigma\right) \text {. }
$$

Thus, for a given jaw separation, the potential energy of the part as a function of jaw positions on the part perimeter is a two-dimensional surface embedded in three dimensions. The potential energy is nonnegative. When the jaws are relaxed (separation is greater than undeformed separation for a contracting grasp) the potential energy is zero. When both jaws are in contact, the potential energy is a quadratic function of the deformation. Equilibria lie at the local minima of this surface. The deform-closure grasp will lie in a strict local minimum. An escape path will start from this point and end at another local minimum with maximum potential energy at a saddle point in the "valley" containing the deform-closure.

Recall that we computed $e_{\max }$, the maximum strain for a unit reduction of jaw separation from the undeformed configuration with the contacts at $n_{0}$ and $n_{l}$. We use this to determine $\sigma_{L}$ as $\sigma_{0}\left(n_{0}, n_{l}\right)-\left(e_{L} / e_{\max }\right)$. The additional potential energy needed for the contacts to squeeze the part to the elastic limit is given by $U_{L}(\sigma)=1 / 2 \mathrm{k}_{01}\left(\sigma_{L}^{2}-\sigma^{2}\right)$. Recall that the quality metric is given by:

$$
Q(\sigma)=\min \left\{U_{A}(\sigma), U_{L}(\sigma)\right\} \text {. }
$$

\subsection{Computing Sample Points}

In this section, we will examine the nature of the function $U_{A}(\sigma)$ to choose appropriate sampling points that determine $\sigma^{*}$ exactly.

Consider a path from $v\left(n_{a}, n_{b}\right)$ to an equilibrium vertex. The path is a sequence of vertices. Each graph vertex $v\left(n_{c}, n_{d}\right)$ on the path has a potential energy function $U\left(n_{c}, n_{d}, \sigma\right)$. The potential energy needed to release the part through this path is given by the upper envelope of all these potential energy functions. There are many equilibrium nodes, and many paths to each equilibrium node. Each path has an upper envelope that gives the potential energy needed. If we consider all the upper envelopes over all the paths and take their lower envelope, this gives the minimum potential energy needed to release the part as a function of contact separation. The difference between this lower envelope and function $U\left(n_{0}, n_{1}, \sigma\right)$ is the threshold potential energy $U_{A}(\sigma)$ of the deform-closure grasp.

Thus, $U_{A}(\sigma)$ is a continuous piecewise quadratic function. Each piece corresponds to the potential energy of one graph vertex (escape configuration). We identify these intervals as follows: we consider every pair of graph vertices and evaluate the values $\sigma_{n}$ of separation for which the potential energy curves of this pair intersect with each other. We then sort these values of $\sigma_{n}$ in ascending order. Between any consecutive pair of $\sigma_{n}$ values in the sorted list, $U_{A}(\sigma)$ will be a quadratic function. We determine $U_{A}(\sigma)$ exactly in each of these intervals by evaluating it at any three points in the interval. We choose the endpoints and the midpoints of the intervals for this purpose. Thus, we evaluate $U_{A}(\sigma)$ exactly in every interval. To maximize $Q(\sigma)$ globally, we maximize it in each interval using the procedure given in the next section and take the largest of these maxima. 


\subsection{Computing Optimal Jaw Separation}

We now consider a given fixed value $\sigma_{i}$ of contact separation, we need to find the threshold potential energy $U_{A}\left(\sigma_{i}\right)$. Consider a path (a sequence of connected graph vertices) from $v\left(n_{0}, n_{1}\right)$ to equilibrium configuration $v\left(n_{a}, n_{b}\right)$. Let $U_{\max }$ be the maximum potential energy over all nodes traversed by this path. There are many equilibrium vertices and many such paths. Let $U_{p}$ be the minimum of the $U_{\max }$ values over all such paths. The threshold potential energy $U_{A}\left(\sigma_{i}\right)$ is given by $U_{p}-U\left(n_{0}, n_{l}, \sigma_{l}\right)$.

We determine $U_{A}\left(\sigma_{i}\right)$ using the contact graph $G$ by an algorithm inspired by Dijkstra's shortest path algorithm. We maintain a set $\Omega$ of vertices with known minimum work needed to get to, and a list $U_{\min }$ of these minimum works. We also compute a list $\Psi$ of estimated minima of work needed to reach vertices in $V$ - $\Omega$ that are adjacent to vertices in $\Omega$. The estimate assumes that in the path used to reach the node, the previous node lies in $\Omega$. We then update $\Omega$ by adding the graph vertex with minimum $\Psi$.

Sub-procedure $U_{A}\left(\sigma_{l}\right)$ :

$$
\begin{aligned}
& \text { Initialize } \Omega \leftarrow\left\{v\left(n_{0}, n_{1}\right)\right\}, U_{\min }\left(v\left(n_{0}, n_{1}\right)\right)=0, \\
& \Psi\left(v\left(n_{a}, n_{b}\right)\right)=U\left(n_{0}, n_{1}, \sigma_{l}\right)-U\left(n_{0}, n_{1}, \sigma_{l}\right) \\
& \quad \text { for all vertices } v\left(n_{a}, n_{b}\right) \text { adjacent to } v\left(n_{0}, n_{1}\right), \\
& \Psi\left(v\left(n_{a}, n_{b}\right)\right)=\infty \text { for others. } \\
& \text { While } \Omega \neq V \text { do } \\
& v\left(n_{c}, n_{d}\right) \leftarrow \arg \min \left\{\Psi\left(v\left(n_{a}, n_{b}\right)\right)\right\} \\
& \Omega \leftarrow \Omega \cup\left\{v\left(n_{c}, n_{d}\right)\right\} \\
& U_{\min }\left(v\left(n_{c}, n_{d}\right)\right)=\Psi\left(v\left(n_{c}, n_{d}\right)\right) \\
& \text { If } v\left(n_{c}, n_{d}\right) \text { is an equilibrium vertex, } \\
& \quad \text { return } U_{\min }\left(v\left(n_{c}, n_{d}\right)\right) \text { and stop. } \\
& \text { For each } v\left(n_{a}, n_{b}\right) \text { in } V-\Omega \text { adjacent to } v\left(n_{c}, n_{d}\right), \text { update: } \\
& \quad \Psi\left(v\left(n_{a}, n_{b}\right)\right) \leftarrow \min \left\{\operatorname { m a x } \left\{\Psi\left(v\left(n_{c}, n_{d}\right)\right),\right.\right. \\
& \left.\left.\quad U\left(n_{a}, n_{b}, \sigma_{l}\right)-U\left(n_{0}, n_{1}, \sigma_{l}\right)\right\}, \Psi\left(v\left(n_{a}, n_{b}\right)\right)\right\}
\end{aligned}
$$

End while.

We can prove that $U_{\min }$ always contains the minimum increase in potential needed to reach any element in $\Omega$ as follows: Initially, when $\Omega=\left\{v\left(n_{0}, n_{1}\right)\right\}$, this is true. When a vertex $v\left(n_{c}, n_{d}\right)$ is added to $\Omega$, assume that $U_{\min }$ is larger than the minimum work needed to reach $v\left(n_{c}, n_{d}\right)$. This implies that in the path that needs to be taken to reach $v\left(n_{c}, n_{d}\right)$ to minimize the additional potential energy needed, the graph vertex preceding $v\left(n_{c}, n_{d}\right)$ is in $V-\Omega$. In the sequence of graph vertices represented by the path, consider the first graph vertex $v\left(n_{a}, n_{b}\right)$ in $V-\Omega$. $\Psi\left(v\left(n_{a}, n_{b}\right)\right) \geq \Psi\left(v\left(n_{c}, n_{d}\right)\right)$ since we add smaller estimates to $\Omega$ first. Thus, $U\left(v\left(n_{c}, n_{d}\right)\right) \geq \Psi\left(v\left(n_{c}, n_{d}\right)\right)$. This contradicts the assumption that $U_{\min }\left(v\left(n_{c}, n_{d}\right)\right)$ is not the minimum possible.

Thus, we obtain $U_{A}\left(\sigma_{l}\right)$ as the difference in the maximum potential energy needed to reach a second equilibrium configuration and the potential energy of the grasp configuration (note that this returns zero for grasp configurations that are not stable equilibria). $U_{L}\left(\sigma_{l}\right)$ is obtained as discussed in the previous section. We thus obtain $Q\left(\sigma_{i}\right)=\min \left\{U_{A}\left(\sigma_{i}\right), U_{L}\left(\sigma_{i}\right)\right\}$. For each interval defined by consecutive values in $\sigma_{n}$, we determine the $U_{A}$ for each endpoint and the midpoint, and hence determine it as an exact quadratic function of $\sigma$. Thus, for each interval, we determine the maximum value of $Q(\sigma)$. We return the value of $\sigma$ for which $Q(\sigma)$ is the global maximum, as $\sigma *$. 


\subsection{Approximation to Optimal Jaw Separation}

In the previous section, we sampled the curve $Q(\sigma)$ at selected values of $\sigma$ to determine the exact function. In this section we give a more efficient algorithm that finds an approximation $\sigma_{\varepsilon}$ to $\sigma *$. We specify $\varepsilon$ as the maximum allowed error in $Q\left(\sigma_{\varepsilon}\right)$ due to the approximation.

In this algorithm, we ignore the sequence $\sigma_{n}$. Instead, we uniformly sampling the interval $\left[0, \sigma_{0}\left(n_{0}, n_{l}\right)\right]$ to maximize $Q(\sigma)$. We define $\lambda=\max \left\{k_{a b} \sigma_{0}(a, b)\right\}$ over all perimeter mesh nodes a, b. With uniform sampling at intervals of size $\xi=\varepsilon / \lambda$, we can show using the derivative of $Q(\sigma)$ that the error in the optimal metric is at most $\varepsilon$.

For all $\sigma_{i}=i \varepsilon / \lambda, \mathrm{i}=0, \ldots,\left\lceil\left(\sigma_{0}\left(n_{0}, n_{1}\right) \lambda / \varepsilon\right)\right\rceil$, we find the threshold potential energy using the procedure described in the previous section. We take $\sigma_{\varepsilon}=\arg \_\max \left\{Q\left(\sigma_{i}\right)\right\}$.

\subsection{Numerical Example}

As shown in Fig. 6, we implement both algorithms and compare them using a sample part with dimensions approximately $12 \mathrm{~mm}$ x $20 \mathrm{~mm}$. The part material is rubber with the standard Young's modulus of $2 \mathrm{MPa}$, and Poisson ratio of 0.1 . The strain at the elastic limit is 0.8 . The undeformed distance between the mesh nodes shown is $10 \mathrm{~mm}$. We modeled the part using I-deas and ANSYS on a PC with a $1.4 \mathrm{GHz}$ Pentium IV processor, running Windows XP. Each FEM problem was solved in approximately 0.28 seconds. The initial computation of all $k_{a b}$ values required 1023 seconds.

The exact algorithm computed the optimal jaw separation to be $5.638 \mathrm{~mm}$ after 9486 iterations (759 seconds).

The approximate algorithm with $\varepsilon=6.46$ Joules (chosen for sample length $\xi=0.1 \mathrm{~mm}$ ) computed the approximate jaw separation to be $5.600 \mathrm{~mm}$ after 44 iterations (3.5 seconds), a speedup of over 200x.

\section{Discussion}

\subsection{Complexity}

As shown by [Ramamurti98], FEM systems can be solved exactly in $O\left(n^{3}\right)$ time for a part with $n$ mesh nodes. For a part with $n$ mesh nodes and $p$ perimeter nodes, the graph used for the algorithm has $O\left(p^{2}\right)$ nodes and $O\left(p^{2}\right)$ edges. Thus, pre-computing the $k_{a b}$ values for each graph vertex requires $O\left(n^{3} p^{2}\right)$ time. Computing $U_{A}$ for a known separation requires $O\left(p^{2} \log p\right)$ time when the graph is implemented as a binary heap (this complexity is identical to that of Dijkstra's algorithm for sparse graphs). For the exact algorithm, there are $O\left(p^{4}\right)$ values of $\sigma_{n}$, and hence $O\left(p^{4}\right)$ intervals to evaluate $U_{A}(\sigma)$. In the approximate algorithm, the distance between sampling points is $\varepsilon / \lambda$, implying that there are $\left(\sigma_{0}(0,1) \lambda / \varepsilon\right)$ sampling points. Thus, the complexity of the exact algorithm is $O\left(n^{3} p^{2}+p^{6} \log p\right)$ while $\sigma_{\varepsilon}$ can be computed in $O\left(n^{3} p^{2}+p^{2} / \varepsilon \log p\right)$ time.

\subsection{Symmetries in D-Space}

If we were to construct a model of D-space by brute force, the amount of computation involved in determining $D_{\text {free }}$ may be extremely high. For example, for the 12 node mesh in Figure 1, the D-Space will be 24-dimensional. However, we can simplify the computation of computing $D_{\text {free }}$ using the symmetries of D-obstacles and of $D_{T}$. D-obstacles are symmetric, since any two triangles of the mesh collide with any given finger body in identical configurations of the triangles. Hence, for every triangle in the mesh, the slice of the D-obstacle (with other nodes at fixed positions) will be identical to that of any other triangle, 
and further, independent of the positions of nodes outside the triangle. Thus, the D-obstacle is defined by the union of prisms whose cross sections are these slices. At the center of all these prisms is the configuration where the part shrinks to a point inside the obstacle. For the part in Figure 1, this causes the D-obstacles to have a 12-fold symmetry due to presence of 12 elements in the mesh. Further, for identical obstacles, each D-obstacle is a copy of every other obstacle displaced by a known vector.

Although the set of topology preserving mesh configurations $D_{T}$ may not be symmetric in itself, we consider a superset $D^{\prime}{ }_{T}$ of $D_{T}$ that consists of all meshes such that no mesh triangles intersect and no mesh triangle has zero area. $D^{\prime}$ contains configurations that cannot be attained by the physical part (such as a mirror image of the undeformed mesh), but $D_{T}$ is a maximal continuous subset of $D_{T}^{\prime}$. For the part in Figure 1, this results in 23 symmetries because of pairs of distinct triangles, 29 symmetries for pairs of triangles with 2 distinct vertices each, and 13 symmetries for pairs of triangles that have 1 distinct vertex each.

\subsection{Conclusion and Future Work}

We presented a new framework and a definition for workholding of deformable parts. We then formulated the problem of optimizing the separation between a pair of jaws holding a deformable polygonal part. We gave a fast approximate algorithm and an exact algorithm to solve this problem.

We are working on reducing the complexity of the exact algorithm by modifying the algorithm to find $U_{A}$ for a given separation to update the estimates for threshold potential energy and optimal separation simultaneously. We believe that there is a much faster algorithm possible since there can be at most $O\left(p^{2}\right)$ escape configurations since there are at most $O\left(p^{2}\right)$ graph vertices. Hence, there can be at most $O\left(p^{2}\right)$ quadratic pieces of $Q(\sigma)$ as against the $O\left(p^{4}\right)$ candidate intervals we consider. Thus, it may be possible to obtain an algorithm that runs in $O\left(n^{3} p^{2}+p^{4} \log p\right)$ time or faster. It may be possible to use Boundary Element Methods [Ramamurti98] to obtain a faster algorithm.

In future research we will generalize the D-Space framework and Deform Closure definition to nontriangular meshes with non-linear FEM, and to three-dimensional parts. We are also working on the mechanics on achieving deform-closure grasps and the necessary and sufficient number of contacts needed.

\section{Acknowledgements}

This work was supported in part by the Ford Motor Company URP 2000-403R and NSF Award DMI0010069. We thank Frank van der Stappen, Ben Wegbreit, Rama Koganti, Matt Zaluzec, Ron Alterovitz, Dezhen Song and Vladlen Koltun for their valuable comments.

\section{References}

[Bayazit02] O. B. Bayazit, J.-M. Lien, N. M. Amato, Probabilistic Roadmap Motion Planning for Deformable Objects, Proc. IEEE Int. Conf. Robot. Autom. (ICRA), Washington, D.C., May 2002.

[Bicchi00] A. Bicchi and Vijay Kumar, Robotic Grasping and Contact: A Review, Proceedings of IEEE International Conference on Robotics and Automation, pp348-353, 2000.

[Brost96] R.C.Brost and K.Y.Goldberg, A complete algorithm for designing planar fixtures using modular components, IEEE Trans. On Robotics and Automation, vol. 12, no. 1, pp. 31 - 46, 1996.

[Cai96] Cai W., Hu S.J., Yuan J.X., Deformable sheet metal fixturing: principles, algorithms, and simulations. Transactions of the ASME. Journal of Manufacturing Science and Engineering, vol.118, (no.3), ASME, p.318-24, Aug. 1996.

[Cheong02] Cheong, J.-S., Goldberg K., Overmars M.H. and van der Stappen, A.F., Fixturing Hinged Polygons, Proceedings, IEEE International Conference on Robotics and Automation, pp. 876-881, 2002.

[Cheong03] Cheong J.-S., Haverkort H.J., van der Stappen A.F., On computing all immobilizing grasps of a simple polygon with few contacts, Proc. of the 14th Annual International Symposium on Algorithms and Computation (ISAAC) (2003), LNCS 2906, Springer Verlag, pp. 260-269, Berlin (2003).

[Corman01] T.H. Corman., C.E. Leiserson. and R.L. Rivest, Introduction to Algorithms, MIT Press, 2001. 
[Doulgeri04] Doulgeri, Z. and Peltekis, J., Modeling and Dual Arm Manipulation of a Flexible Object, IEEE International Conference on Robotics and Automation, April 2004.

[Gopalakrishnan02] K. "Gopal" Gopalakrishnan, Ken Goldberg, Gripping parts at concave vertices, IEEE International Conference on Robotics and Automation, 2002, Page(s): 1590 -1596, Volume: 2, 2002.

[Gopalakrishnan03] K. "Gopal" Gopalakrishnan, Matthew Zaluzec, Rama Koganti, Patricia Deneszczuk and Ken Goldberg, "Unilateral" Fixturing of Sheet Metal Parts Using Modular Jaws with Plane-Cone Contacts, Proceedings, IEEE International Conference on Robotics and Automation, pp 3953-3958, 2003.

[Gopalakrishnan04] K. "Gopal" Gopalakrishnan and Ken Goldberg, D-Space and Deform Closure: A Framework for Holding Deformable Parts, IEEE International Conference on Robotics and Automation (ICRA), May 2004.

[Gopalakrishnan04b] K. "Gopal" Gopalakrishnan and Ken Goldberg, Computing Deform Closure Grasps, $6{ }^{\text {th }}$ International Workshop on the Algorithmic Foundations of Robotics, Utrecht/Zeist, The Netherlands, July, 2004.

[Gopalakrishnan04c] K. "Gopal" Gopalakrishnan, Ken Goldberg, Gary M. Bone, Matthew Zaluzec, Rama Koganti, Rich Pearson, Patricia Deneszczuk, Unilateral Fixtures for Sheet Metal Parts with Holes, IEEE Transactions on Automation Science and Engineering, second issue, vol. 1, no. 2, pp.110-120, 2004.

[Guibas99] Guibas, L.J.; Holleman, C.; Kavraki, L.E.; A probabilistic roadmap planner for flexible objects with a workspace medial-axis-based sampling approach, Proceedings, IEEE/RSJ International Conference on Intelligent Robots and Systems, Volume: 1, Page(s): 254 -259, 17-21, Oct. 1999.

[Heinrich00]Heinrich D. and Worn H., editors, Robot manipulation of deformable objects, Springer Verlag, May 2000.

[Hirai01] Hirai S., Tsuboi T., Wada T., Robust Grasping Manipulation of Deformable Objects, Proc. IEEE Symposium on Assembly and Task Planning, pp.411-416, May 2001.

[Holleman98] Holleman, C.; Kavraki, L.E.; Warren, J., Planning paths for a flexible surface patch, Robotics and Automation, 1998. Proceedings. 1998 IEEE International Conference on, Volume: 1, Page(s): 21 -26, 16-20 May 1998.

[Howard97] Howard, A.M. and Bekey, G.A., Recursive Learning for Deformable Object Manipulation, 8th International Conference on Advanced Robotics, pp. 939-944, Monterey, CA, July 7-9, 1997.

[Jia04] Jia, Y.-B., Computation on paramtric curves with an application in grasping, International Journal of Robotics Research, Vol. 23, No. 7-8, pp. 825-855, 2004. 2004.

[Kim03] Byoung-Ho Kim, Shinichi Hirai, and Takahiro Inoue, Analysis on the Fundamental Deformation Effect of Soft Fingertips for Soft-fingered Object Manipulations, Proc. IEEE/RSJ Int. Conf. on Intelligent Robots and Systems, Vol.4, pp.3698-3704, Las Vegas, October, 2003.

[Lamiraux99] Lamiraux, F.; Kavraki, L.E.; Path planning for elastic plates under manipulation constraints, Robotics and Automation, 1999. Proceedings. 1999 IEEE International Conference on, Volume: 1, Page(s): 151 -156, 10-15 May 1999.

[Li02] Li B., Shiu B.W., Lau K.J., Fixture configuration design for sheet metal assembly with laser welding: a case study. International Journal of Advanced Manufacturing Technology, vol.19, (no.7), Springer-Verlag, p.501-9, 2002.

[Li02b] Li H. F., Ceglarek D., Shi J., A Dexterous Part-Holding Model for Handling Compliant Sheet Metal Parts, ASME Transactions, Journal of Manufacturing Science and Engineering. Vol. 124, No. 1, pp. 109-118, 2002.

[Lin00] Lin, Q., Burdick, J.W., Rimon, E., A stiffness-based quality measure for compliant grasps and fixtures, IEEE Transactions on Robotics and Automation, volume: 16, issue: 6, pages:675 - 688, Dec. 2000.

[Markenscoff90] X. Markenscoff, L. Ni and C. H. Papadimitriou, The Geometry of Grasping, International Journal of Robotics Research, Vol. 9, No. 1, pp 61-74, 1990.

[Mason01] Mason M.T., Mechanics of Robotic Manipulation, MIT Press, 2001.

[Menassa91] Menassa R., De Vries W., Optimization Methods Applied to Selecting Support Positions in Fixture Design, ASME Journal of Engineering for Industry, vol 113, pp. 412-418, 1991.

[Milgram02] Milgram, R.J. and Trinkle, J.C., The Geometry of Configuration Spaces for Closed Chains in Two and Three Dimensions, Homology, Homotopy and Applications, International Press, 2002.

[Mishra87] B. Mishra, J. Schwarz, and M. Sharir, On the existence and Synthesis of Multifinger Positive Grips, Algorithmica 2, 1987.

[Moll04] Moll, M. and Kavraki L., Path Planning for Minimal Energy Curves of Constant Length, Proceedings, IEEE International Conference on Robotics and Automation, 2004.

[Ramamurti98] Ramamurti V., Computer Aided Mechanical Design and Analysis, McGraw Hill, July 1998.

[Reuleaux1876] F. Reuleaux, The Kinematics of Machinery. New York: Macmillan 1876, republished by New York: Dover, 1963.

[Rimon96] Rimon E. and Burdick J., On force and form closure for multiple finger grasps, Proceedings of IEEE International Conference on Robotics and Automation, pp. 1795 -1800 vol.2, 1996.

[Rimon98] Elon Rimon and Joel Burdick, Mobility of bodies in contact - I, IEEE transactions on Robotics and Automation, 14(5): 696-708, 1998.

[Rimon99] Rimon, E. and Blake, A., Caging planar bodies by one-parameter two fingered gripping systems, International Journal of Robotics Research, v18, n3, pp. 299-318, March 1999.

[Rong99] Rong, Y. and Zhu, Y., Computer-aided Fixture Design, Marcel Dekker, ISBN: 0-8247-9961-5, New York, 1999.

[Salisbury82] Salisbury, J.K. Kinematics and Force Analysis of Articulated Hands. Ph.D. Thesis, Stanford University, 1982. 
[Selig00] J. M. Selig. Geometrical Foundations of Robotics. World Scientific, 2000.

[Shiu97]Shiu, B.W., Ceglarek, D., Shi, J., Flexible Beam-Based Modeling of Sheet Metal Assembly for Dimensional Control, Trans. of NAMRI, Vol. XXV, pp. 49-54, 1997.

[Shiu03] Shiu, B.W., Apley, D., Ceglarek, D., Shi, J., 2003 Tolerance Allocation for Sheet Metal Assembly using Beam-Based Model, Trans. of IIE, Design and Manufacturing, , Vol. 35, No. 4, pp. 329-342, April 2003.

[Somoff1900] P. Somoff, Uber gebiete von schraubengeschwindigkeiten eines starren korpers bieverschiedener zahl von stuz achen, Zeitschrift fur Mathematic and Physik, vol. 45, pp. 245-306, 1900.

[vanderStappen99] Van der Stappen A.F., Wentink C., Overmars M.H., Computing form-closure configurations, Proceedings of IEEE International Conference on Robotics and Automation, vol.3, pp. 1837 -1842, 1999.

[Wakamatsu96] Wakamatsu, H., Hirai, S., and Iwata, K., Static Analysis of Deformable Object Grasping Based on Bounded Force Closure, Proc. IEEE Int. Conf. on Robotics and Automation, Vol.4, pp.3324-3329, Minneapolis, April, 1996.

[Zhu03] Zhu X., Ding H., Wang J., Grasp analysis and synthesis based on a new quantitative measure, IEEE Transactions on Robotics and Automation, vol 19, no. 6, pp. 942-953, Dec 2003. 


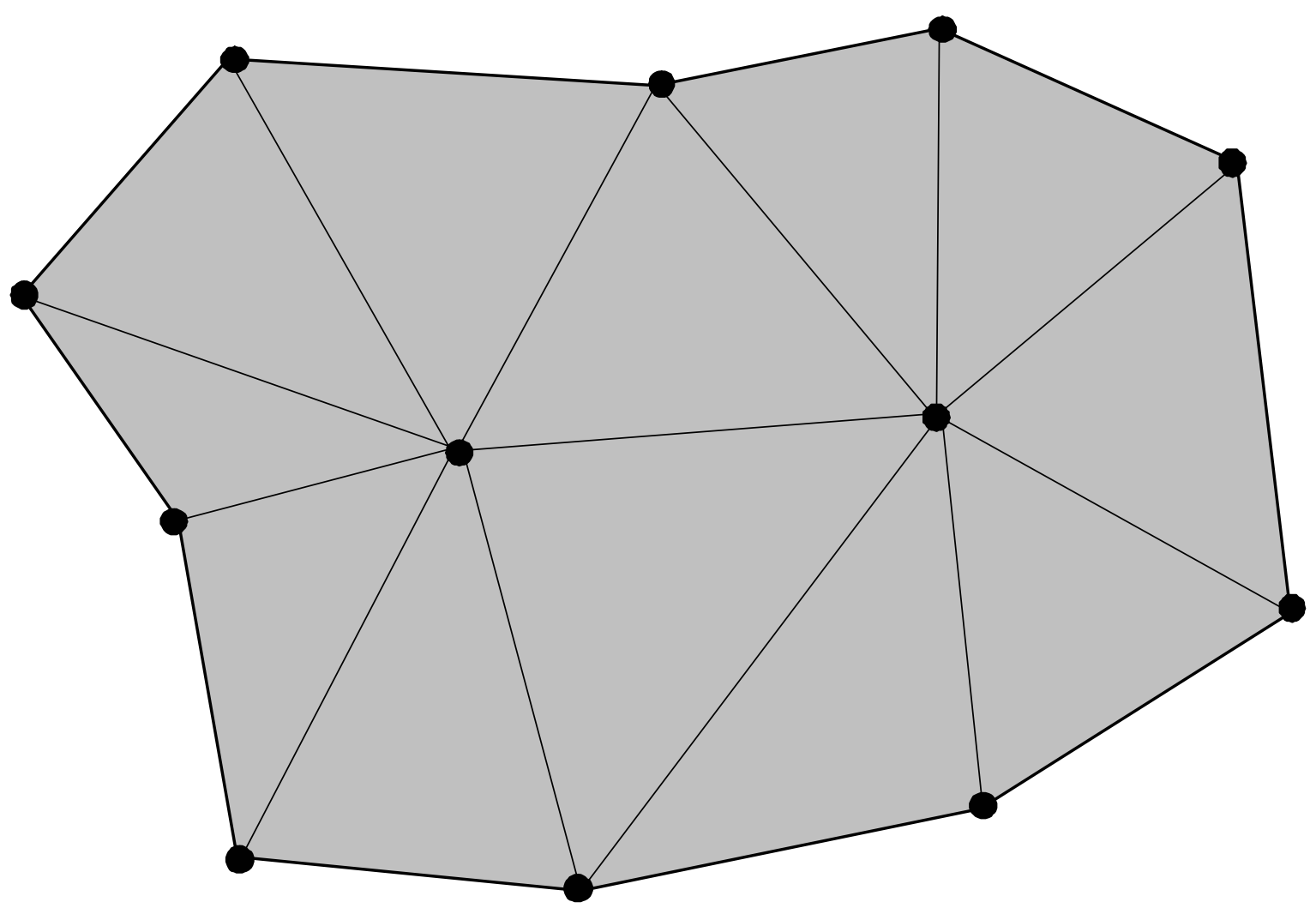

(a)

Page 16 of 23 


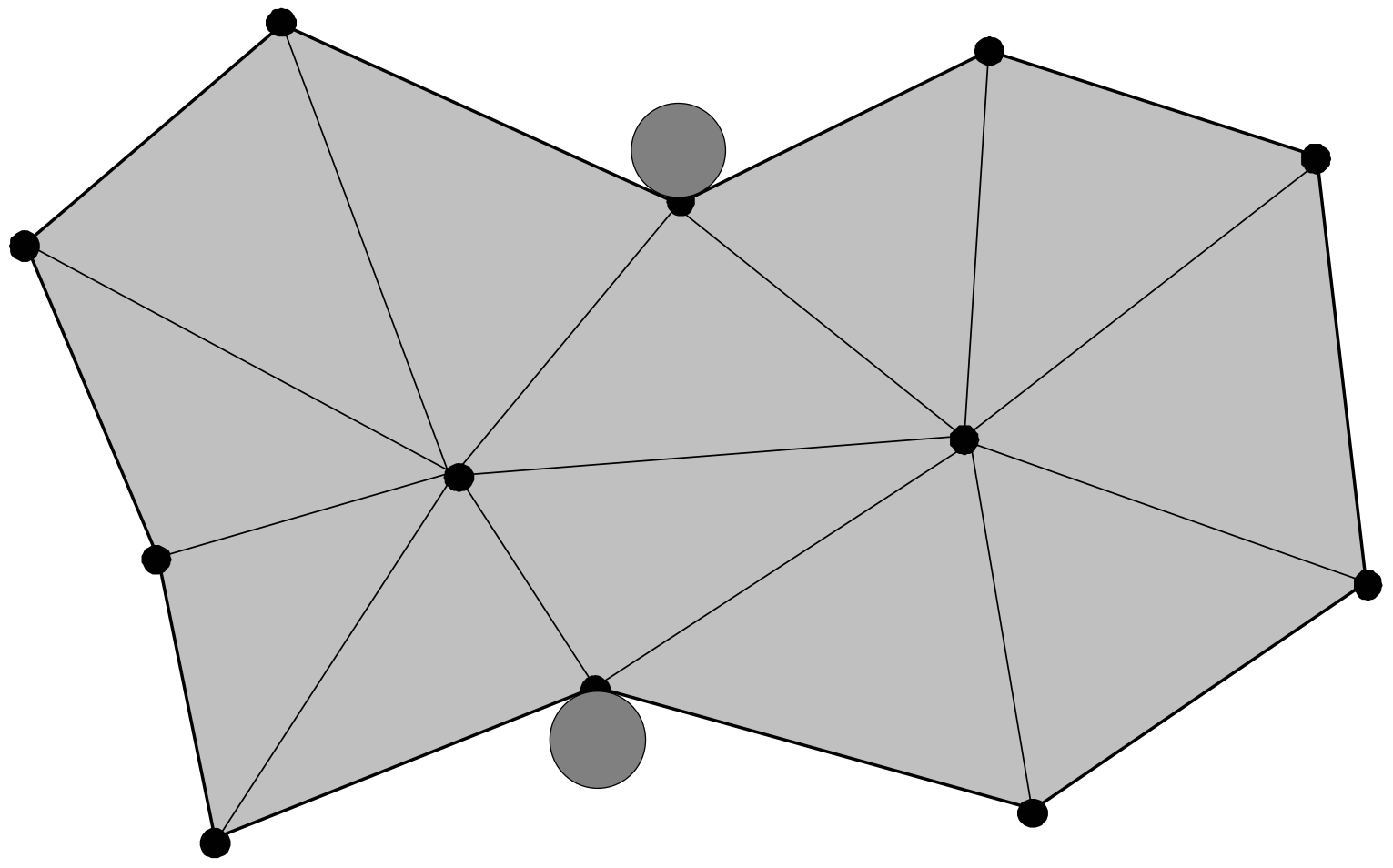

(b)

Fig. 1. (a) Deformable part, modeled as a polygon with a finite element mesh and given stiffness matrix. The part perimeter behaves like a planar structure whose perimeter is composed of struts of variable length, with hinges at each vertex. (b) The same part held in deform closure by two frictionless point contacts. 


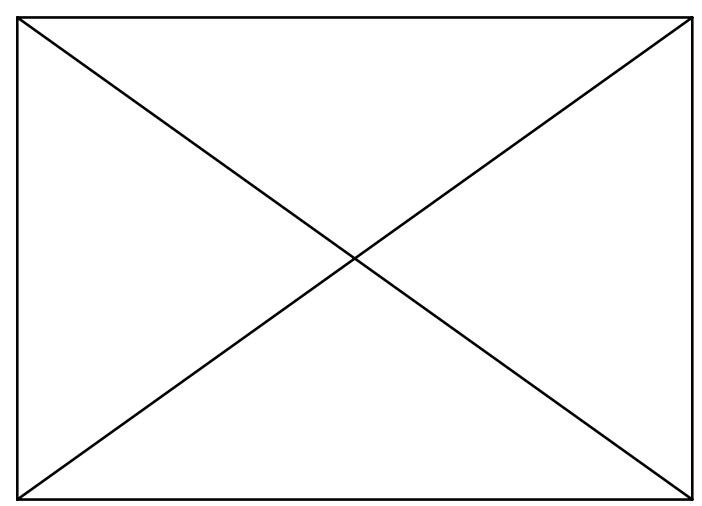

(a)

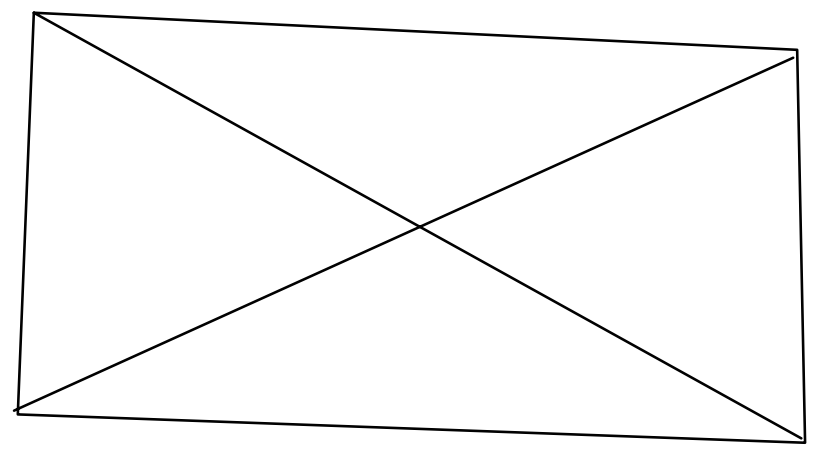

(b)

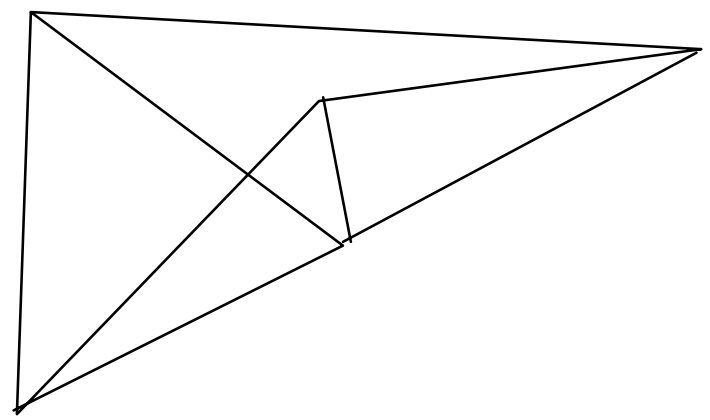

(c)

Fig. 2. For the rectangular part in (a), deformed configuration (b) lies in $D_{T}$, while deformed configuration (c) lies in $D_{T}^{C}$. 


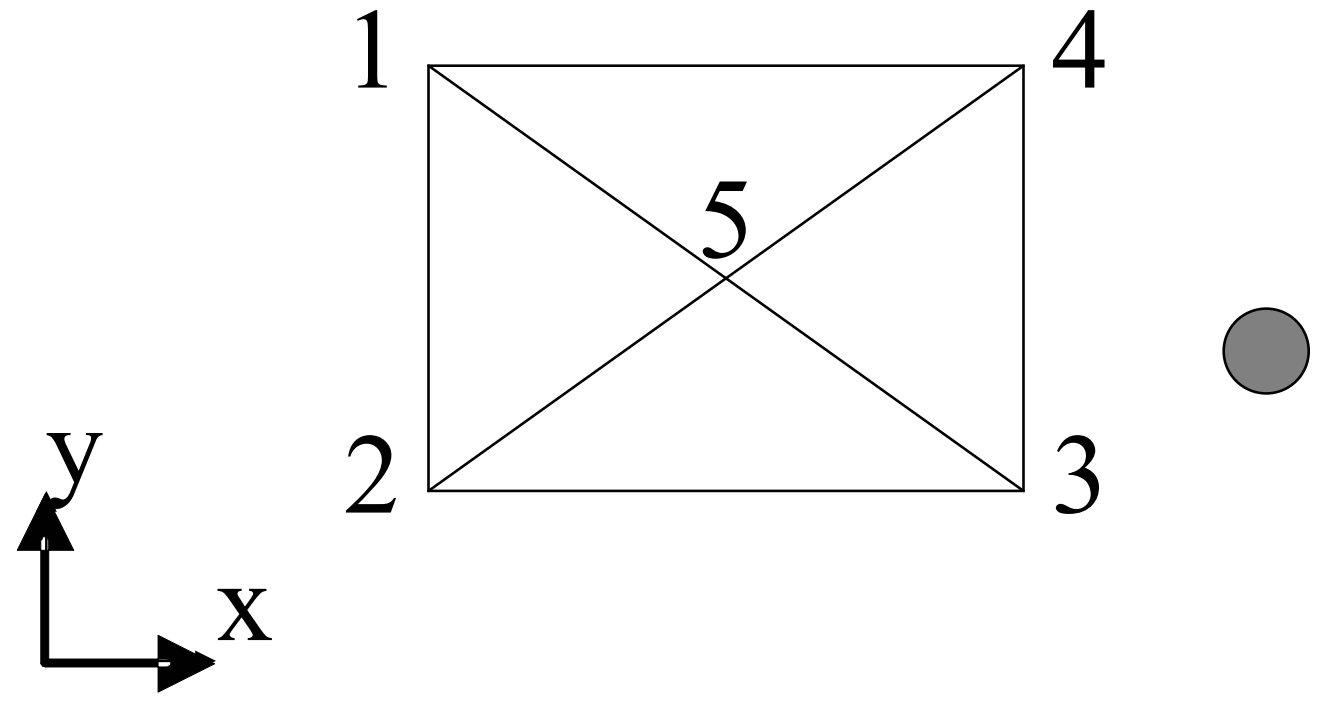

(a)
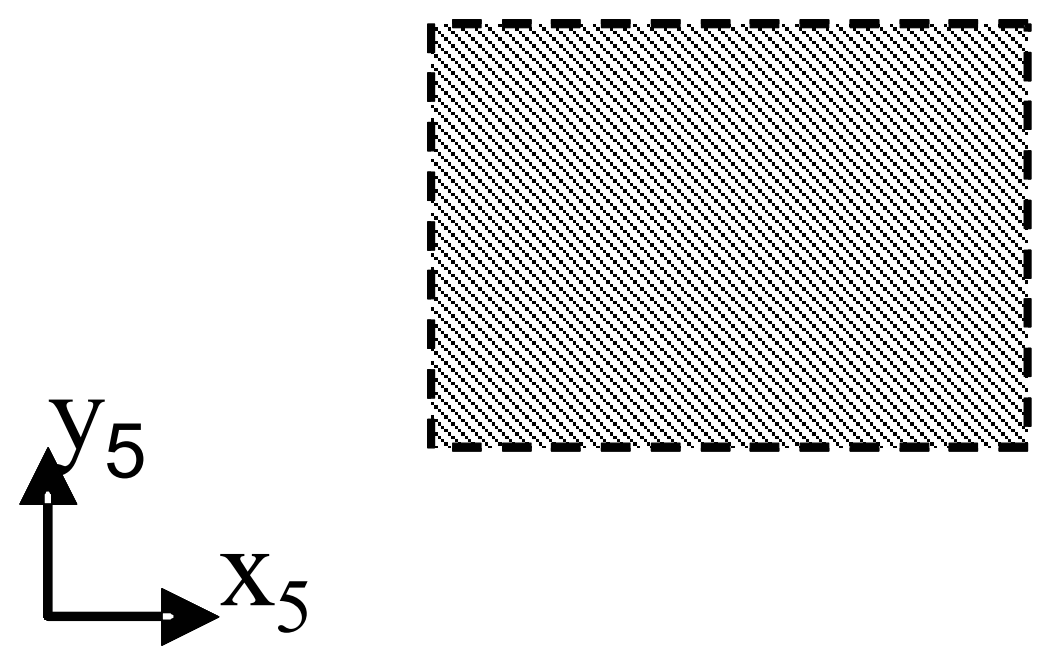

(b)

Page 19 of 23 


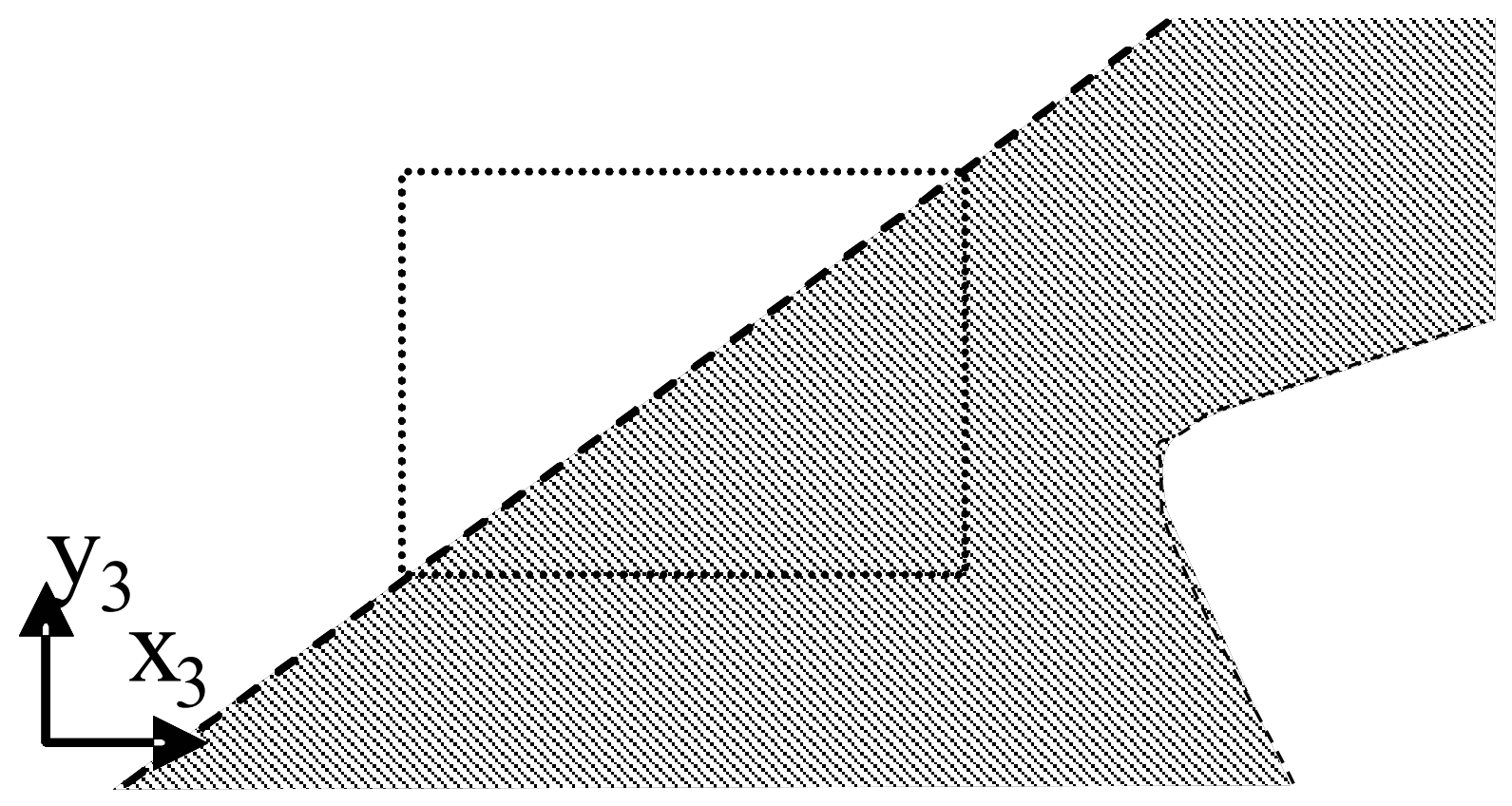

(c)

Fig. 3. For an example part with 5 nodes and one circular obstacle, $D_{\text {free }}$ for nodes 5 and 3 ((b) and (c) respectively) shown when the remaining nodes of the rectangle are held fixed in the positions shown in (a). The shaded region lies inside $D_{\text {free }}$. 


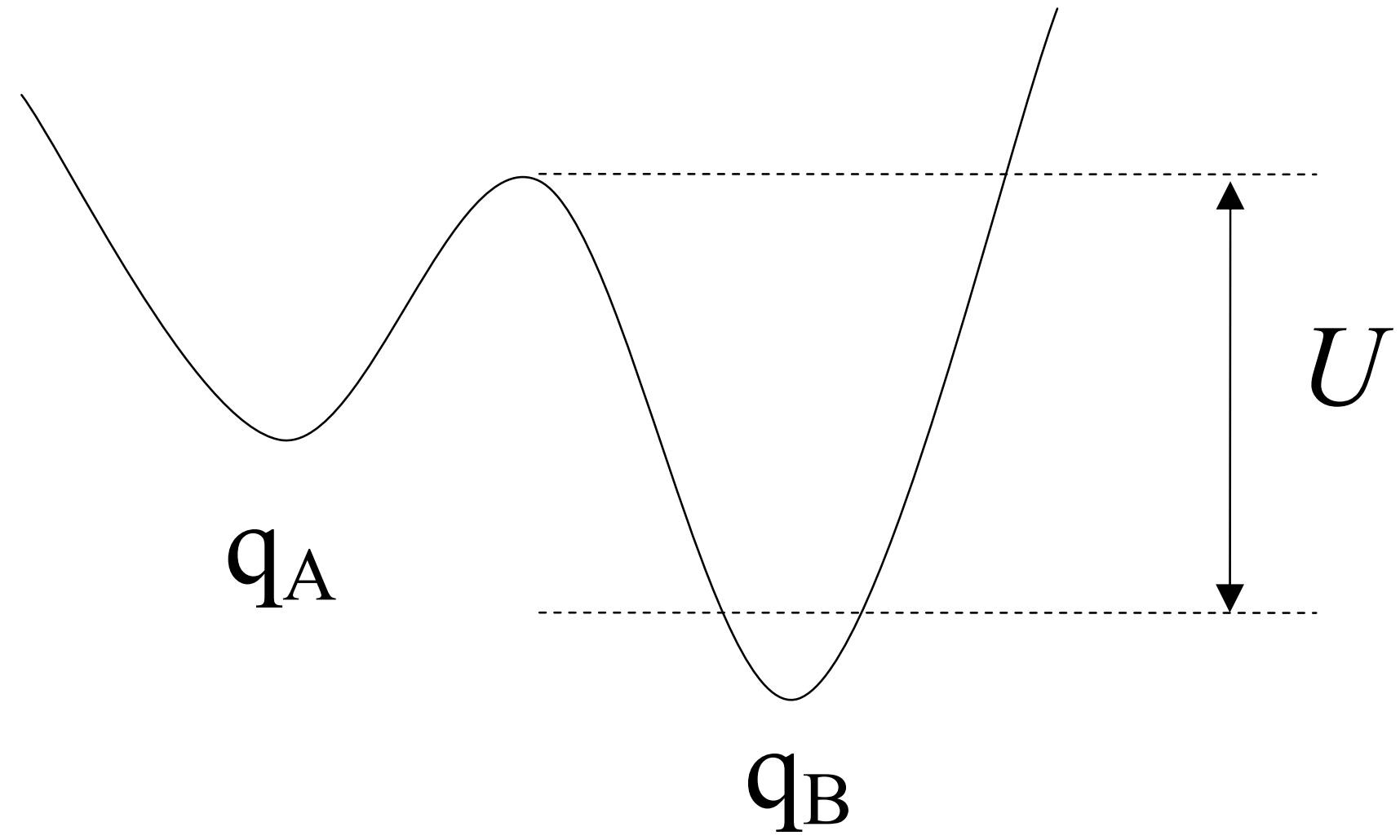

Fig. 4. For the Potential Energy curve shown, $q_{A}$ and $q_{B}$ are stable equilibria. But for the shown value of $U$, only $q_{B}$ satisfies stable (U). 


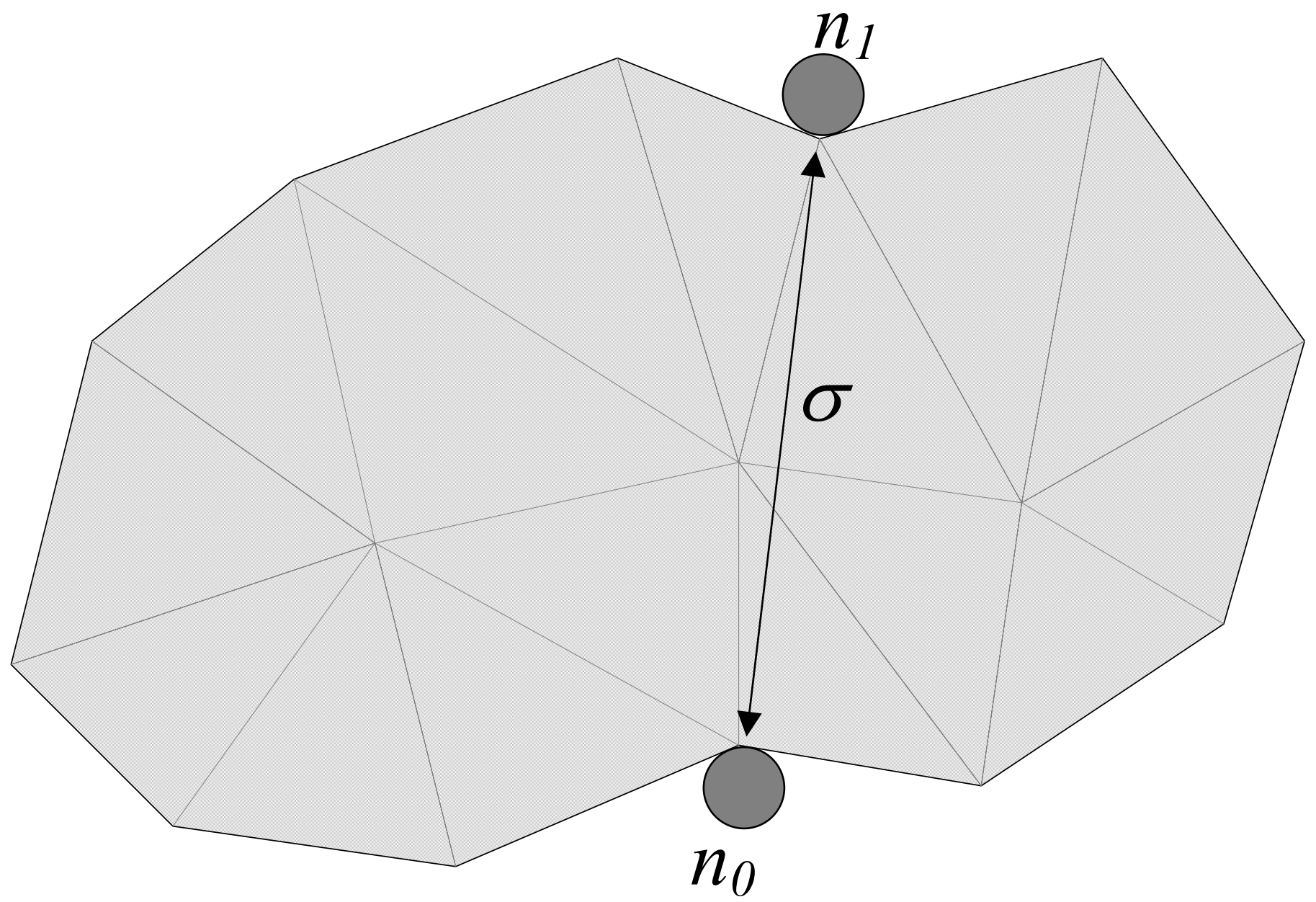

Fig. 5. The algorithm maximizes quality metric $Q(\sigma)$, where $\sigma$ is the contact separation. We want to find $\sigma^{*}$, the jaw separation that balances the energy needed to release the part vs. that needed to plastically deform it. 


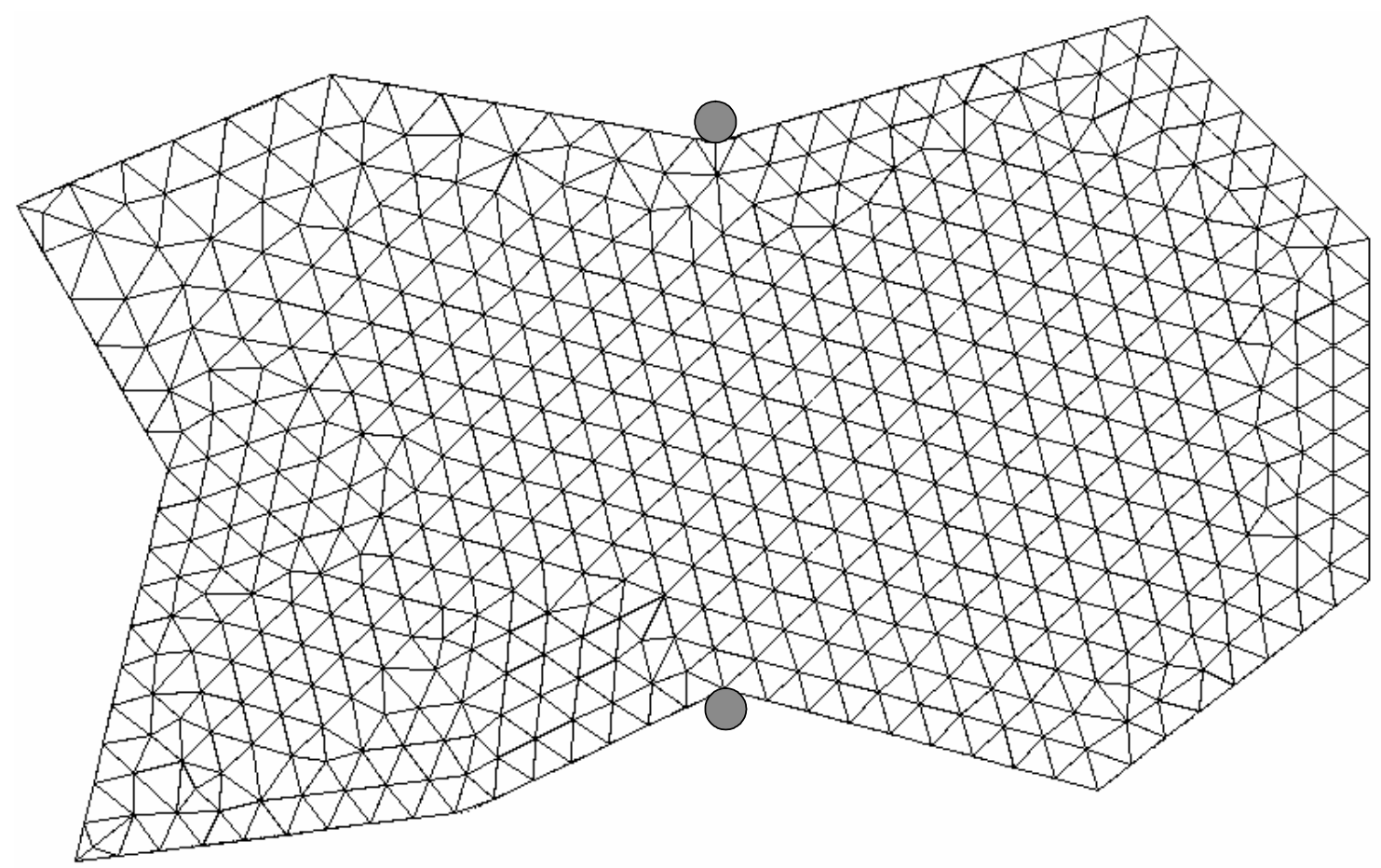

(a)

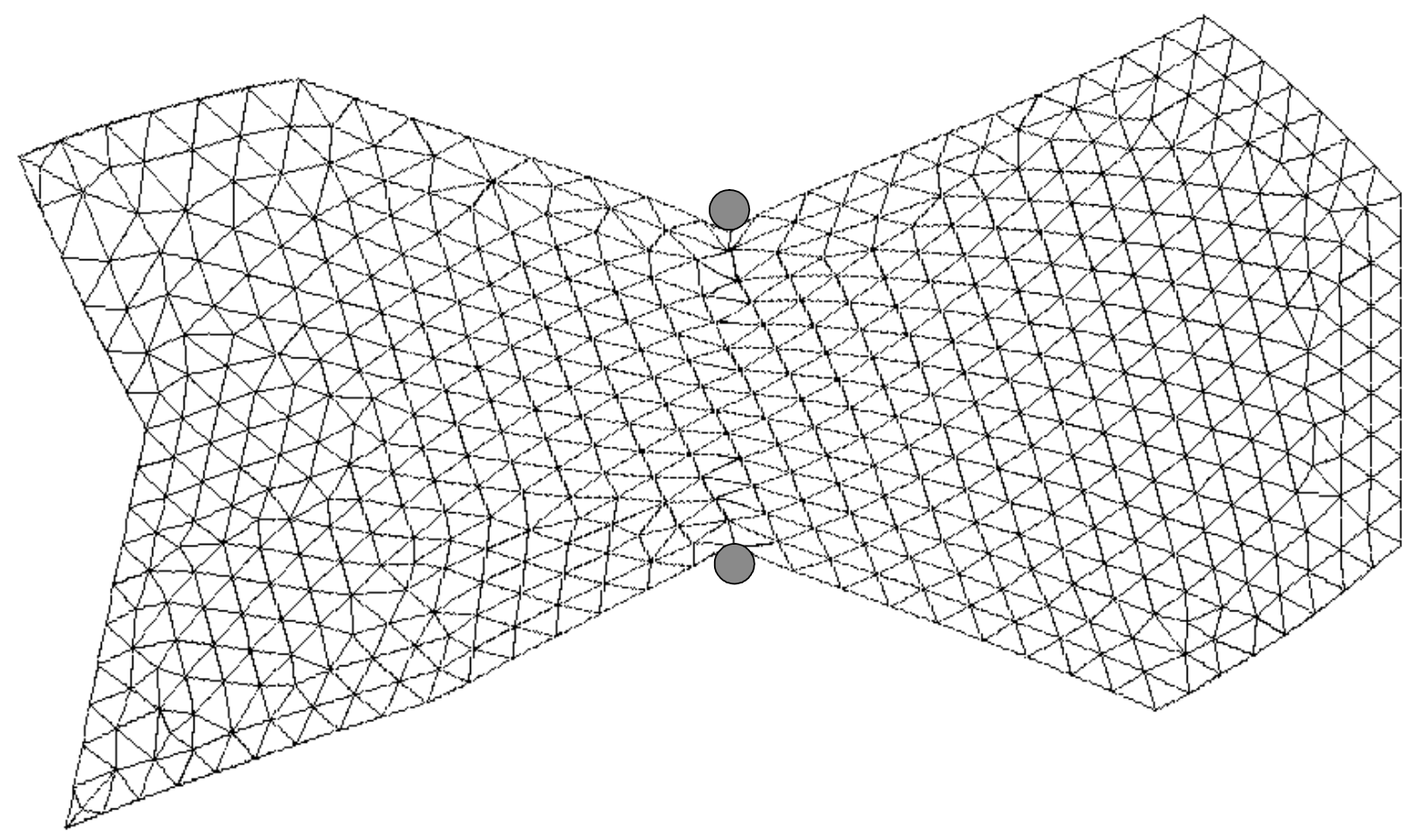

(b)

Fig. 6. (a) Example part with initial jaw separation $10 \mathrm{~mm}$ for the perimeter mesh nodes shown. (b) The same part at optimal jaw separation $5.638 \mathrm{~mm}$.

Page 23 of 23 